\title{
Revascularization of post-traumatic leg amputation: a case report
}

\section{Isaac Okyere ${ }^{1}$, Vincent Ativor ${ }^{2}$ and Perditer Okyere ${ }^{3}$}

Ghana Med J 2019; 53(4): 308-311 doi: http://dx.doi.org/10.4314/gmj.v53i4.10

\author{
${ }^{1}$ Cardiovascular and Thoracic Surgery Unit, Department of Surgery, School of Medicine and Dentistry, College \\ of Health Sciences, Kwame Nkrumah University of Science and Technology and Komfo Anokye Teaching \\ Hospital, Kumasi, Ghana \\ ${ }^{2}$ Trauma and Orthopaedic Surgery Unit, Department of Surgery, School of Medicine and Dentistry, College of \\ Health Sciences, Kwame Nkrumah University of Science and Technology and Komfo Anokye Teaching Hospi- \\ tal, Kumasi, Ghana \\ ${ }^{3}$ Departement of Internal Medicine, School of Medicine and Dentistry, College of Health Sciences, Kwame \\ Nkrumah University of Science and Technology and Komfo Anokye Teaching Hospital, Kumasi, Ghana
}

Corresponding author: Dr Isaac Okyere

E-mail: drokyere@yahoo.com

Conflict of interest: None declared

\section{SUMMARY}

There are few reports on lower extremity revascularization because of its high risks of general and local complications as well as poor functional prognosis. However, revascularization of the traumatically amputated lower extremity is a technically feasible surgical undertaking if there can be effective collaboration between the orthopaedic surgeon and the vascular surgeon. Successful outcome is usually judged by functional achievements of the patient toward returning to the preinjury level. Appropriate patient selection significantly increases the potential for obtaining a satisfactory outcome. We report the successful revascularization of a near amputation of the right leg of a young man who was knocked down accidentally by a speeding taxi leading to mangled and near amputation of his right leg. He underwent successful revascularization and currently doing well, one year after the surgery. Successful revascularization is possible if indicated in less resource countries especially if there is an experienced team of vascular and orthopaedic surgeons.

Keywords: Revascularization, leg, trauma, amputation, repair Funding: None

\section{INTRODUCTION}

Revascularization of the traumatically amputated lower extremity is a technically feasible surgical undertaking. Successful outcome must be judged by the functional achievements of the patient toward returning to the preinjury level. Appropriate patient selection significantly increases the potential for obtaining a satisfactory outcome. Patients in whom peripheral nerve injury precludes return of sensation in the extremity or in whom severe joint destruction will yield an immobile extremity will have marginal results. The risks of revascularization, including blood transfusions, sepsis, and prolonged hospitalization, must be weighted heavily against prosthetic substitution as an alternative. However, replantation successfully done provides a superior functional and cosmetic outcome for the patient. Revascularization must preserve adequate functional length and the durability of the extremity must always be considered. Even in low middle-income countries with less human resource, an excellent result can be achieved if there can be effective collaboration between the vascular surgeon and the trauma orthopaedic surgeon.

\section{CASE REPORT}

Twenty-one year old with no known chronic illness was an un-helmeted motorbike rider who was knocked down by a speeding taxi .The impact was mainly his right leg which was trapped by the fender of the car resulting in near amputation of the right leg and just partly hanging by the Achilles tendon and bleeding profusely .He was immediately rushed to the Accident and the Emergency Centre of the Komfo Anokye Teaching Hospital in Kumasi, Ghana for management. He was conscious and alert and well orientated on admission with Heart rate of 111 beats/minute, Blood Pressure of 114/70 mmHg, Respiratory cycle of 26 breaths /minute with $\mathrm{SPO}_{2}$ of $98 \%$ on room air. His temperature was $35.7^{\circ} \mathrm{C}$ with a random sugar level of $9.2 \mathrm{mmol} / 1$. 


\section{Case Report}

On examination of the leg, there was near amputation of the right leg which was barely hanging by Achilles tendon with bleeding.

The right foot was insensate and cold, with absent dorsalis and posterior tibialis artery pulsations. There was completely displaced transverse fracture of the distal tibia and fibula (Figure 1). The MESS score (Mangled Extremity Severity Score) was 7. (2 for Skeletal or soft tissue injury: medium energy - multiple fractures; 3 for Limb ischemia: 1 for Systolic BP > $90 \mathrm{~mm} \mathrm{Hg}$; 1 for Age $<30$ years). The injury was classified as type $\mathrm{C}$ and type IV as per Gustilo and Byrd open tibial fracture classification respectively. Other systems were normal. The extended focused assessment with sonography in trauma (eFAST) was negative for haemoperitoneum, haemopericardium and haemopneumothorax. He was quickly resuscitated with Intravenous fluids, anti-tetanus prophylaxis, antibiotics and blood was taken for grouping and crossmatching against four units of blood. Urgent blood investigation showed an $\mathrm{Hb}$ of $4.5 \mathrm{~g} / \mathrm{dl}$, white cell count of $12.02 \times 10^{9} / 1$, and platelets level of $237 \times 10^{9} / 1$. Renal function was within normal limits. He was then rushed to theatre with consent taken for examination under anaesthesia and possible revascularization or amputation. Under spinal anaesthesia, supine position and aseptic conditions, there was a Grade IIIC Open fracture of the distal third of the right tibia and fibula with near amputation, the wound was moderately contaminated with moderately crushed soft tissue at the fracture ends.

The posterior tibialis artery could not be visualized but both the anterior tibialis artery and greater saphenous vein were all completely transected, thrombosed and retracted, however the Achilles tendon was intact. Wound debridement and copious saline irrigation of the wound and the amputation stumps was done. The tibia and fibula were reduced by $7 \mathrm{~cm}$ to healthy margins and the fracture ends were opposed, reduced and anule spanning external fixator applied. The ends of the transected greater saphenous vein were dissected free from the surrounding tissue, the edges freshened, and a size 3 FG Fogarty embolectomy catheter passed, and clots removed to ensure adequate inflow and backflow. It was then flushed with a 500 IU heparin saline. This was followed with an end-toend repair with prolene $6 / 0$ in a continuous single layer fashion. The anterior tibialis artery which was thrombosed was also dissected free from the surrounding muscles, securing adequate proximal and distal ends and the edges were also freshened and a size 3 FG Fogarty embolectomy catheter was passed both proximally and distally through to remove clots. Also flushed with $500 \mathrm{IU}$ heparin saline flush to ensure effective inflow and backflow. The artery was then repaired in a single continuous end-to-end anastomosis with prolene $6 / 0$.
The foot became warm immediately after the anastomosis with adequate colour change. Capillary refill was less than 3 seconds on table. The muscles were apposed gently with vicryl $2 / 0$ and the wound was further irrigated with warn saline. A vacuum drain was placed, and the skin was closed in an interrupted fashion with nylon $2 / 0$.

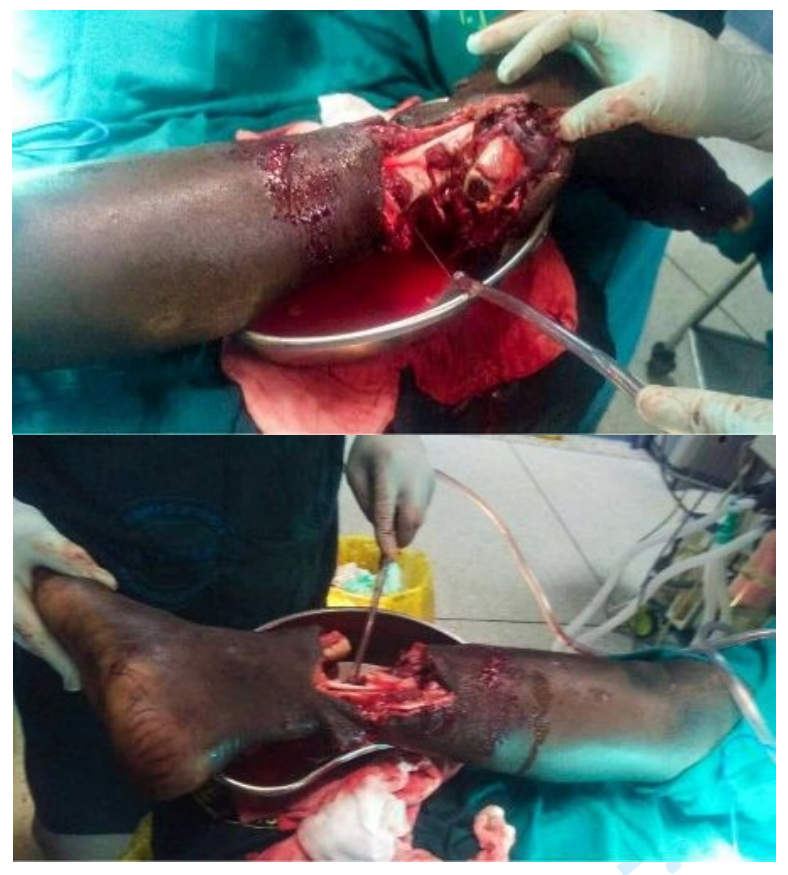

Figure 1 Showing the mangled right foot.

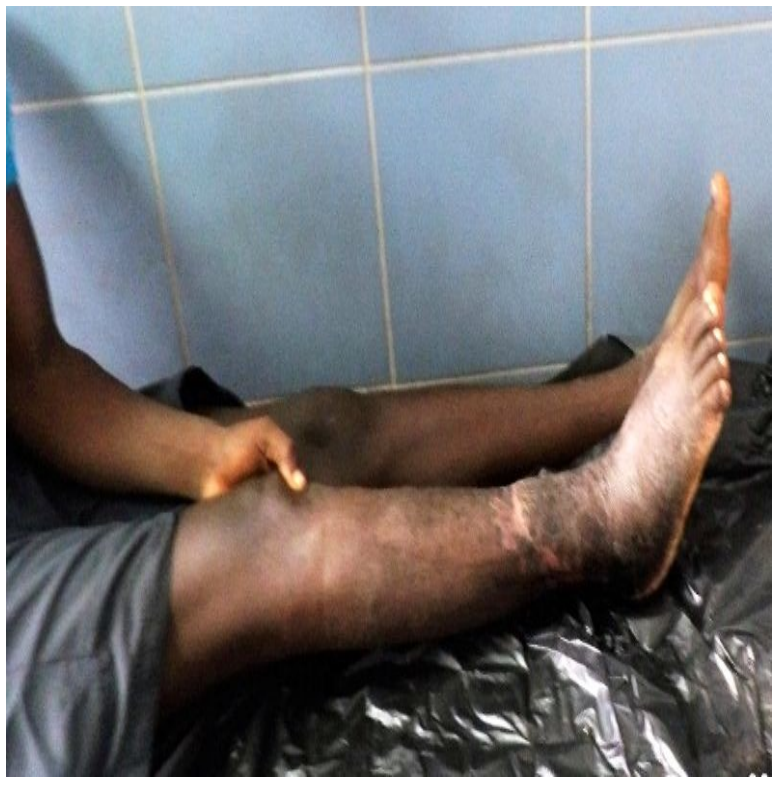

Figure 2 Three months post operatively, showing the shortened limb with healed wound. 
The pin sites were dressed and bandaged. He had prophylactic anticoagulation with low molecular weight heparin, Enoxaparin 40mg daily for seven days postoperatively and was discharged home twenty-five days after surgery. The ischemic time from injury to revascularization was 5 hours. A total of 4 units of red blood cells was transfused intraoperatively and another two units of blood postoperatively. Figure 2 shows the state of the limb at three months. One year after the accident he has good function and good sensibility in the sole of his foot. Crutches were used for 6 months without weight bearing and thereafter partial weight-bearing was allowed.

\section{DISCUSSION}

The common cause of lower limb injury is usually road traffic accidents. ${ }^{1}$ The management of traumatic amputation of lower limbs even if brought early to hospital is either completion amputation especially in less resource centres and middle income countries like ours or replantation or revascularization if there is indication for salvage surgery and there is local expertise. However amputation is 3 times more costly than salvaging procedures. $^{2}$

The salvage procedure of the lower limb from replantation or revascularization has limitations especially when shortening of the limb is done which is required for a tension-free vascular anastomosis. Limb shortening affects posture and function ${ }^{3}$ The first macro-replantations had low success rate because the initial wound debridement was limited in order to restrict inequality in length between the lower limbs. ${ }^{4,5}$

A $7 \mathrm{~cm}$ shortening of the leg was done for our patient and this might have contributed to the successful take of the revascularization. As noted by Joon et al, ${ }^{2}$ the primary goals of lower limb injury management especially for replantation or revascularization are the maintenance of a well vascularized extremity, skeletal continuity and lastly the presence of a protective sensation with the intact innervation to the plantar surface of the foot. ${ }^{2}$ Therefore in replantation or revascularization the presence of at least one main arterial supply, one venous drainage and an intact posterior nerve are mandatory for success. This, we were able to achieve for our patient in whom we repaired the anterior tibialis artery, greater saphenous vein and the intact posterior nerve was helpful.

Moreover the main criteria to decide for replantation of an amputated limb includes the patients age and the general condition, the ischemic time and the level of injury, type and extent of tissue damage which has various classifications such as the Byrd and the Gustilo Grading criteria. ${ }^{6}$ People over the age of 50 years generally have greater number of complications, and the worst prognosis. Associated multiple lesions (head trauma, internal organ lesions), state of shock, cardiac problems or other diseases such as diabetes etc., are contraindications of replantation or revascularization. For the ischemic time, even in the presence of good preservation of the distal segment (cold ischemia), replantation time must not exceed 6 hours if segments contain large muscular masses. ${ }^{7}$ This not only guarantees limb survival but also avoids severe post-operative complications such as cardiac or renal failure. The extent of injury is classified according to the MESS Score with scores less than 5 resulting in good outcomes. ${ }^{8}$ Though it must be stated that the decision to amputate or re-implant or revascularize a limb should be individualized. Our case was a young man who was stable on admission, not in shock, and happened to have been brought to the hospital within 4 hours of the injury and therefore qualifying for a successful revascularization or salvage procedure. The results of various large series studies show that lower limb replantation is still worthwhile even in less resource centres like ours, as advocated by Hierner et al. ${ }^{9}$ Hence every effort should be put in place to salvage legs if possible, especially when there is indication and there is availability of local expertise.

\section{CONCLUSION}

Successful revascularization or replantation of a mangled limb is possible, if indicated, in less resourced countries. The success of the procedure would require an experienced team of vascular and orthopaedic surgeons.

\section{ACKNOWLEDGEMENT}

We are grateful to both the surgical theatre team at the accident and emergency centre of Komfo Anokye Teaching Hospital, Kumasi, Ghana and to the patient for consenting for the publication of the case.

\section{REFERENCES}

1. Rajput DU, Powar RS, Sulhyan SR. Replantation of post-traumatic lower limb amputation.

Int J Pharma Med Biol Sci. 2014; 3(1):46-51.

2. Song D. Reconstructive Surgery: Lower Extremity Coverage. In: Hong JP, ed. Reconstructive Surgery. 3rd ed. Philadelphia; 2013:127-150.

3. Salon A, Liverneaux PA, Dubert T, Bleton R, Alnot JY. Long-term review of five leg replantations: Emergency strategy and examples of lengthening of the leg on nerve regeneration. Injury. 2006; 37(9):869-876. doi:10.1016/j.injury.2006.06.004

4. Fukui A, Tamai S. Present status of replantation in Japan. Microsurgery.1994;15(12):842847

doi:10.1002/micr.1920151204 


\section{Case Report}

5. Zhang J, Chen ZW. Retrospective of the replantation of severed limbs in the People's Republic of China: Current status and prospects. Microsurgery. 2002; 22(1):39-43. doi:10.1002/micr.22007

6. Gayle LB, Lineaweaver WC, Buncke GM, Oliva A, Alpert BS, Billys JB BH. Lower extremity replantation. Clin Plast Surg. 1991; 18(3):437-447.

7. Bueno RA, Battiston B, Ciclamini D, Titolo P, Panero B, Tos P. Replantation: Current concepts and outcomes. Clin Plast Surg. 2014. doi:10.1016/j.cps.2014.03.010

8. Zhong-Wei C, Meyer VE, Kleinert HE BR. Present indications and contraindications for replantation as reflected by long-term functional results. Orthop Clin North Am. 1981; 12(4):849-870.

9. Hierner R, Berger AK, Frederix RP. Lower leg replantation-Decision-making, treatment, and longterm results. Microsurgery. 2007; 27(5):398-410. doi:10.1002/micr.20380 